\title{
Toward A Paradigm Shift in Cancer Research \& Innovation for Patients in France
} \author{
${ }^{1}$ Coordinator of the Avise Initiative \\ ${ }^{2}$ Montpellier Universities, Europe \\ ${ }^{3}$ Centre Leonberard, Lyon, Europe \\ ${ }^{4}$ Chru lille, Europe \\ ${ }^{5}$ Chru, Strasbourg, Europe \\ ${ }^{6}$ Oncologist, Nancy, Europe \\ ${ }^{7}$ Aphp, Paris, Europe \\ ${ }^{8} \mathrm{Crct}$, Canceropole Grand Sud-Ouest, Europe \\ ${ }^{9}$ Inserm Umr911, Marseille France, Europe \\ ${ }^{10}$ Centre Jean Perrin, Clermont-Ferrand, Europe \\ ${ }^{11} \mathrm{Hcl}$ Lyon, France, Institut De Cancerologie Des Hcl, Universite De Lyon, \\ ${ }^{12}$ Roche Diagnostics, Meylan, Europe \\ ${ }^{13}$ Clinical Operations, Roche France, Boulogne-Billancourt, Europe \\ ${ }^{14}$ Gouvernmentalaffairs, Roche France, Boulogne-Billancourt, Europe \\ ${ }^{15}$ cancéropôle Grand Sud-Ouest, Toulouse, Europe \\ ${ }^{16}$ Institute Roche, Boulogne-Billancourt, Europe
}

C. Long ${ }^{1}$, PA Bonnet ${ }^{2}$, D. Pérol ${ }^{3}$, M. Hebbar ${ }^{4}$, P. Barthélémy ${ }^{5}$, Elisabeth Luporsi ${ }^{6}$, Philippe Saiag ${ }^{7}$, Gilles favre $^{8}$, JosephCiccolini ${ }^{9}$, FrédériquePenault-Llorca ${ }^{10}$, Gilles Salles ${ }^{11}$, Jean-Yves Blay ${ }^{3}$, Gilles Freyer ${ }^{11}$, FrédéricWarnier ${ }^{12}$, FrédéricFleury ${ }^{13}$, Emmanuelle Garault ${ }^{14}$, Cécile Faurie $^{15}$, and Patrice Denèfle*16

Submission: July 13, 2016; Published: August 01, 2017

*Corresponding author: Patrice Denèfle, Institute Roche, for Research and Translational Medicine, France, Europe, Tel: 33147614301; Email: pdenefle@gmail.com

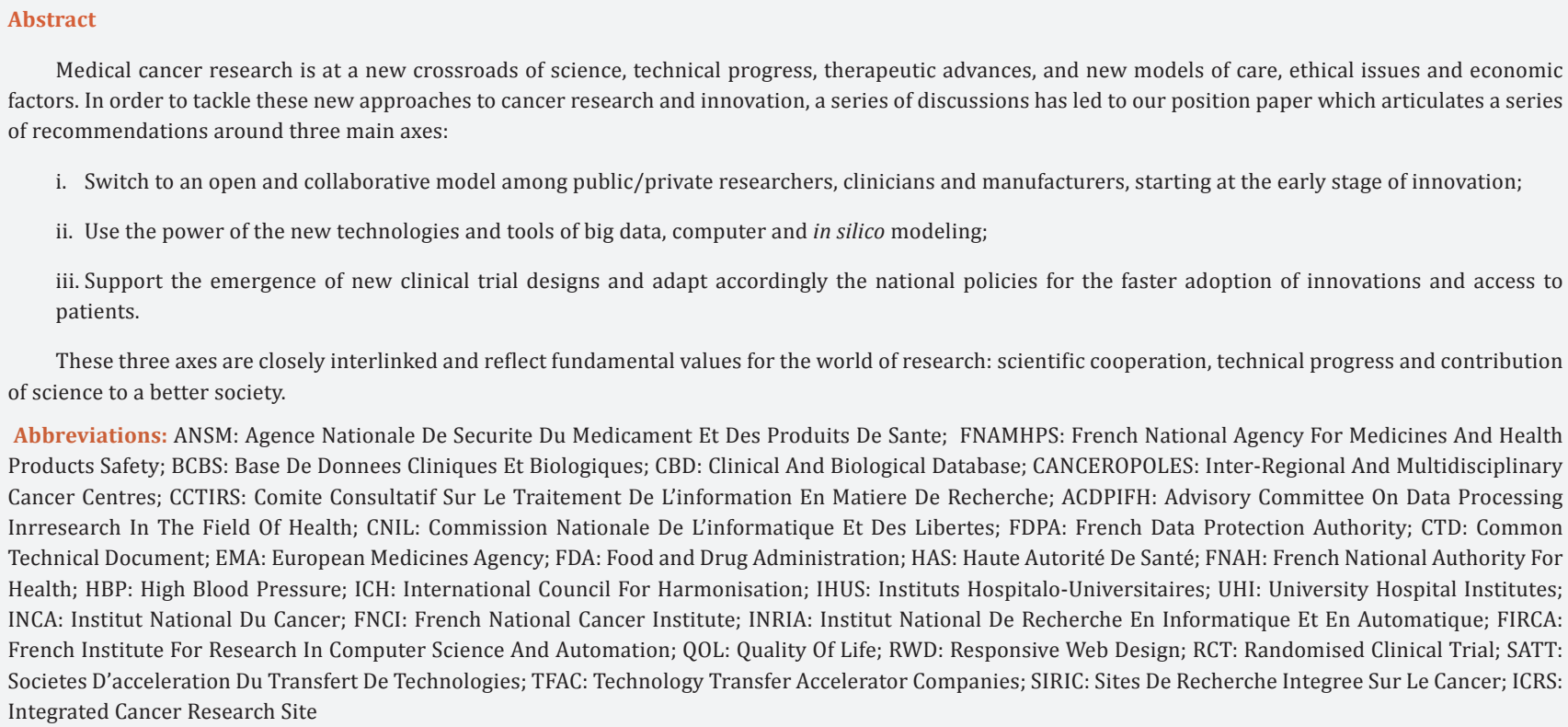

Medical cancer research is at a new crossroads of science, technical progress, therapeutic advances, and new models of care, ethical issues and economic factors. In order to tackle these new approaches to cancer research and innovation, a series of discussions has led to our position paper which articulates a series of recommendations around three main axes:

i. Switch to an open and collaborative model among public/private researchers, clinicians and manufacturers, starting at the early stage of innovation;

ii. Use the power of the new technologies and tools of big data, computer and in silico modeling;

iii. Support the emergence of new clinical trial designs and adapt accordingly the national policies for the faster adoption of innovations and access to patients.

These three axes are closely interlinked and reflect fundamental values for the world of research: scientific cooperation, technical progress and contribution of science to a better society.

Abbreviations: ANSM: Agence Nationale De Securite Du Medicament Et Des Produits De Sante; FNAMHPS: French National Agency For Medicines And Health Products Safety; BCBS: Base De Donnees Cliniques Et Biologiques; CBD: Clinical And Biological Database; CANCEROPOLES: Inter-Regional And Multidisciplinary Cancer Centres; CCTIRS: Comite Consultatif Sur Le Traitement De L'information En Matiere De Recherche; ACDPIFH: Advisory Committee On Data Processing Inrresearch In The Field Of Health; CNIL: Commission Nationale De L'informatique Et Des Libertes; FDPA: French Data Protection Authority; CTD: Common Technical Document; EMA: European Medicines Agency; FDA: Food and Drug Administration; HAS: Haute Autorité De Santé; FNAH: French National Authority For Health; HBP: High Blood Pressure; ICH: International Council For Harmonisation; IHUS: Instituts Hospitalo-Universitaires; UHI: University Hospital Institutes; INCA: Institut National Du Cancer; FNCI: French National Cancer Institute; INRIA: Institut National De Recherche En Informatique Et En Automatique; FIRCA: French Institute For Research In Computer Science And Automation; QOL: Quality Of Life; RWD: Responsive Web Design; RCT: Randomised Clinical Trial; SATT: Societes D'acceleration Du Transfert De Technologies; TFAC: Technology Transfer Accelerator Companies; SIRIC: Sites De Recherche Integree Sur Le Cancer; ICRS: Integrated Cancer Research Site 


\section{Introduction}

The first axis, which advocates a new collaborative research model (named 3.0), refers to this value of scientific cooperation and aims to respond pragmatically to the current challenges such as the need for a permanent trans disciplinary dialogue to raise the level of understanding of the complexity of living things. This is also the meaning of the second axis around "in silico" technologies, which gives new technical means to researchers to go even further in scientific exploration and technical experimentation. The advent of "in silico" in medical research is the new avatar of this digital technological revolution. It opens up the possibility to simulate and visualize concepts which could be handled in the past without going through the real life. As the field of possibilities extends to infinite, in silico approaches will enable researchers to better target their effort and identify the most promising leads, which can result to clinical applications impacting the health status of individuals. In a way, this collaborative research model 3.0 , in combination with in silico approaches, radically transforms the researcher's experience. The researcher of today, passionate, invested, reminds of the figure of Christopher Columbus, audacious enough to start towards the unknown, but adequately accompanied by an entire crew on board in order to face the unexpected. Christopher Columbus was mistaken about his initial scientific undertaking, but his error led to one of Mankind's greatest discoveries. Conversely, tomorrow's researcher is like Thomas Coville, the solitary world sailing record holder. He had gone, sailed and arrived alone at destination. However his great story was indeed a collective adventure as Thomas Coville was fully connected to his team during the World tour. Moreover he prepared his achievement for ten years, with partners from diverse backgrounds, driven by the same sportive, technical and human challenge. Thanks to this collaboration, Thomas Covilles mashed this World record. Looking at these two examples, it is tempting to imagine the great story of Christopher Columbus with the technological means of today [1-5].

History cannot be changed, but it can be repeated. Hence the third axis, on the emergence of new clinical trial designs, which evolve and improve in the light of this new research model 3.0 and in silico. In a way, cancer research is going through a series of learning and integration cycles to translate the scientific and technical progresses into clinical benefits. The same applies to the access to innovation, that is currently based on a model of randomized, phase III therapeutic trials for product registration, while experts believe that this model no longer answers the new research questions asked by the medicine of precision, the knowledge of the tumor microenvironment, the use of real-life data, the therapeutic combinations, etc $[6,7]$.

Modestly but surely, the proposals presented hereafter, resulting from our work, aim to fully integrate biological research and collective intelligence to make use of the full power of emerging technologies and adapt the rules of access to innovations (Figure 1) In our opinion, it is no longer feasible to pursue research and innovation in isolation, and even worse in a closed competition model. Beyond the discourse promoting more cooperation, opening up, networking, convergence, breakdown of silos and core beliefs, our discussions led to draw a path for a more efficient and successful R\&D process, with better sharing of gains and risks in every innovation approach. In order to be able to trace this path and give a direction, a diagnosis was made without the complacency of the current system.

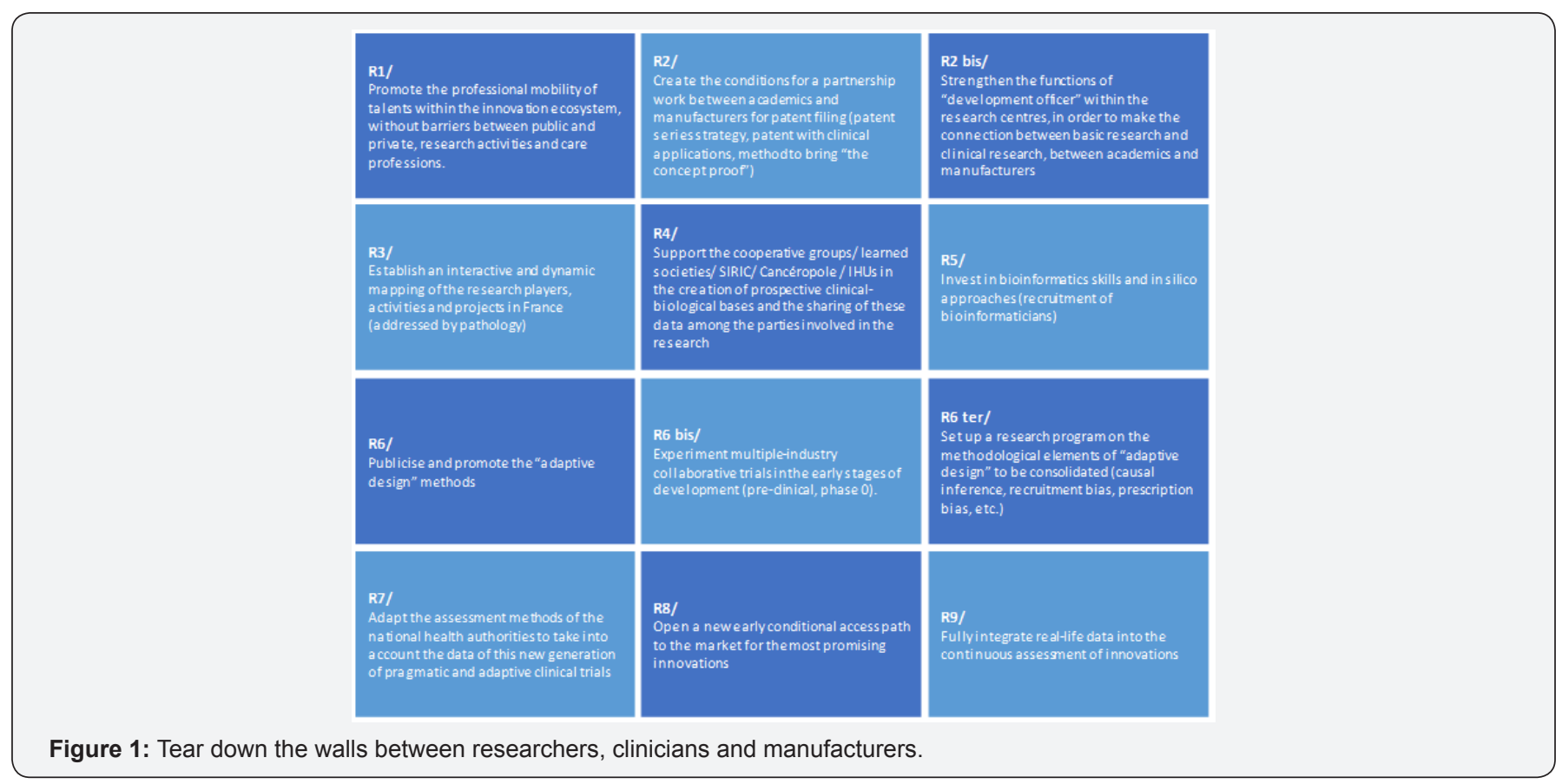


The current and commonly used expression "from bedside to bench to bedside", reflects an approach to translational cancer research, but this vision requires a revision of the point of view on the interactions among the various partners involved in research and innovation. Both researchers in basic and social sciences, clinicians, manufacturers, patients and also managers and funders of the healthcare system should work together. This has been partially achieved up to now albeit in a relay race mode and a linear process instead of running together, where team goal and vision were sometimes unclear, thus resulting in tremendous innovation attrition. The current system may not have been able to generate its own productivity, not relying on entrusted collaboration and cooperation. It allowed frustration, suspicion, mistrust, and misunderstanding among the actors. We believe collectively that this system has lived. At the risk of limiting France to a role of walk-on actor on the world stage, the experts believe that the French system of research and innovation has no choice but to reform and re organise itself in depth. By "tearing down the walls", a new synergistic model would promote open collaborations among researchers, clinicians and manufacturers, enabling organizations and different structures to take part of the same research team, in project mode [8-10].

Several very specific actions are proposed, in order to "tear down the walls".

The first one is obviously to R1/ Promote the professional mobility of talents within the innovation ecosystem, without barriers between public and private, research activities and care professions.

\section{Several approaches should be considered}

I. Promote internships in industry for all medical and pharmacy interns during their initial training course, even for those who do not intend to embrace research carriers. In the same spirit, facilitate the relationships between doctoral students and manufacturers; establish joint post-doctoral and clinical projects, etc. The goal is to provide each researcher or healthcare professional with a real first contact during his initial training, through an experience of collaboration with the industrial world.

II. Encourage individuals to consider double-directional occupational mobility - from the public to the private sector, and reciprocally, from the bedside of the patient to the laboratory, from the lab bench to the hospital. Today, these professional motilities are possible, legally speaking, but marginal in number and may still result in more complex career plans for these atypical pathways. Although research and innovation are part of the collaborative processes, it is nonetheless true that cultures are different and languages are specific to each type of actor. Beyond the simple idea of switching places for a better understanding, these mobility plans would allow people to change their perspective and align vision.
III. Create joint project research teams, in a model of "extended laboratory", where professionals may belong to different structures while contributing to common projects and benefiting from the knowledge ecosystem around the project. Here we would literally find the concept of "tearing down the walls", since researchers would be in the same place, whether this place is physical or virtually integrated. Broader than ever diversity yet complementary sets of expertise and profiles are requested. The complexity of our research in Oncology reinforces the need for cooperation between the players, as no one is really able to cover all skills. The field is indeed getting broader and much more interconnected to other fields that are also scientifically and technically expanding such as in Big Data, molecular genomics, robotisation, systems biology, etc. We believe strongly that pooling of resources, either in a project or in a permanent mode, would greatly facilitate cooperation, since it would pre-empt recurrent contract negotiation since the team is already formed. Thus, in the vision of "tearing down the walls", the approach is to create an organizational environment in which researchers and developers from all sides can work together. Simplification of administrative and contractual processes will generate value and will be economically profitable. This indeed can take various forms but the goal will remain to create a fluid flow of collaborations.

As a natural goal in Innovation is also value-creation, we felt collectively a need for action regarding intellectual property with a proposal aimed to R2/ Create the conditions for a partnership work between academics and manufacturers for patent filing (patent series strategy, patent with clinical applications, method to bring "the concept proof").One of the keys in this public-private collaboration is to create a framework environment that would secure academic researchers, in that they would not have to fear spoliation of innovation by the industry partner. Another key is to develop a method, requiring only a limited investment, and making it possible to qualify the industrial potential (proof of concept) of the innovations revealed by the researchers. It is not realistic to ask academics to provide a level of proof that would be expected by a venture capital, or it would mean that the innovation is no longer at an early stage of development, but with sufficiently maturity to give substance to an industrial project in the medium term. One pilot study that Cancéropole Grand Sud-Ouest and Institute Roche have launched in 2016 in close collaboration is called SYNERGY. This aims at catalyzing early stage scientific interactions between public and private scientists in order to complete scientific proof of concept experiments before intellectual property can be generated. This new type of early stage partnership is clearly a sign of entrusted relationships which pre-figurates the emerging open-innovation era. The pilot is now on and several projects are on their way to emerge. If this pilot is proven successful, we would like to expand to other cancéropôles and industry partners so as to disseminate 
the model. The challenge is to ensure that the academics and Industry can discuss the translational potential of an advance in research before this advance has already yielded results, in which case the research-manufacturer relationship would be more a matter of the trade agreement than of the scientific and technical partnership [11-18].

The gain for both parties of a common reflection on patents is double:

a) select the most promising paths in terms of translational potential, that is which can translate into clinical applications for the patient and reduce the rate of attrition of innovations while avoiding pursuing non-economically or clinically viable paths

b) Improve the economic and commercial operation of patents, with an intellectual property strategy that organises the industrial development of innovation: licensing agreements, royalties. Also include the clinical applications in patents to maintain commercial control of innovations.

One challenge will be to develop a common set of rules between the academic and industrial structures to share risk and economic value of innovation at the time of patent filing or subsequent value-creation based on clear milestones. This new way of risk-sharing in value -creation and project development will require solid know-how and dedicated human resources, within academic research institutions, SIRIC, Cancéropôles, IHUs, etc. This is the main purpose of the following proposal. R2bis/ Strength then the functions of "development officer" within the research centres, in order to make the connection between basic research and clinical research, between academics and manufacturers

The SATT should also have a greater role to play in this translational research, by mobilizing also the scientific advances that at first do not fall directly within the field of cancer care, but which could give rise to applications. For example, this is the case for upstream pharmaco-chemistry work or biomarker research. More Trans disciplinary work needs to emerge as is the case in large competivity poles or in historic centers of excellence where Physics, Mathematics, Biology and Medicine are still effectively connected. Many academic teams in France are internationally recognized for their expertise in Biomedicine, molecular biology, micro environment analysis and genomics, and their work regularly leads to breakthrough innovations. However, these new innovations do not necessarily and obviously find a clinical use, or in any case, the clinical potential is insufficiently validated to allow an industrial project at this early stage. The proposal aims to address this gap between scientific knowledge and industrial development, by focusing on the "start-up" link in the innovation ecosystem.

More generally, one of the shortcomings expressed by the parties involved is that they cannot easily and quickly identify the relevant interlocutors in relation to their research activities. Ultimately, each individual manages on his/her own through informal networking, which they were able to build over the years. As a consequence, innovative projects are slowed down by a lack of professional networking activity. Accelerator programs are needed to provide collective structuring of knowledge and expertise, hence the following proposal, often made by health care and research institutions.

R3/ Establish an interactive and dynamic mapping of the research players, activities and projects in France (addressed by pathology)

This mapping of research expertise and communities has been developed for instance in certain pathologies/organs oncology specialties, such as in sarcoma, renal cancer, skin cancer. At first, these initiatives were initiated by groups of clinicians who were involved in structuring a research community around their pathology. The idea is to capitalize on these successes to expand on this mapping in a progressive way, with the following characteristics:

i. The mapping must include an entry by pathology, organ, network by structure or now also by biomarker

ii. It should remain open to consultation by any researcher or practitioner, even if said practitioner is not concerned with pathology (at first)

iii. It should be interactive, that is, each user must be able to contribute to the mapping: create a sheet, complete a sheet, etc.

iv. It should be dynamic, with regular updates; to do this, an "online" version is prescribed

v. It may include at least three types of content: a presentation sheet for each expert, a presentation sheet of the structures involved in the research, a presentation sheet of the research projects

vi. Beyond these basic functionalities, other usages can be developed, such as contacts, suggestions of contacts, the identification of common subjects between the experts of the base, etc.

The interest of such tool is to connect people and contribute to making sets of expertise or projects more visible, when the experts do not spontaneously know or that even escape their field of reflection for various reasons (competitor laboratory, related topic, lack of time, etc.) It was also stressed that the role of learned societies and cooperative groups in this networking of experts and projects.

This second axis, was also the subject of a strong consensus between the experts of this discussion panels. It is about being able to carry out basic, translational and clinical research work, using new in silico approaches, i.e. without in vivo investigation, 
or rather by repositioning "in vivo" on stages where it will be really necessary.

The possibilities offered by these new technical tools are very broad

A. multiply the trials and tests without the constraints of a standard clinical trial, with zero human risks, but also a more controlled cost of activities

B. re-explore the potential of existing therapies, with "cold tests"

C. identify the targets by modelling, even simulate the effects of a therapy on a virtual population

D. explore the therapeutic combinations, in particular the treatment combinations, in a much easier, simpler and more economical way

E. assess the benefits of therapeutic strategies by treatments such as big data

F. highlight risk factors, factors of loss (gain) of chance for the patients, by comparing medical care

G. allow a prospective approach to research

These in silico approaches, in addition to minimizing the human risk associated with "in vivo" therapeutic trials, bring decisive added value to the new-generation trials in relation to the precision medicine. Indeed, it becomes increasingly difficult to constitute sufficiently robust samples for the trials of targeted therapies such as the family of immune the rapies, whether for phase II trials, and all the more so for phase III trials. In silico allows researchers, from the early stage, in phase 0 or 1 , to model on a much larger virtual population (which is what in vivo currently allows today at the same stage of advancement) and to identify one or multiple targets for the therapeutic innovation? In other words, the target validation would occur much earlier in the research process, leading to a review of phase II and III trials, as conducted with randomized trial methodologies. This section is more particularly developed in the third axis of the design of clinical trials.

Two priority actions were identified on this axis:

a) R4/ Support the cooperative groups/learned societies / SIRIC / Cancéropôle / IHUs in the creation of prospective clinical-biological bases (with contextual individual clinical data) and the sharing of these data among the parties involved in the research

b) $\mathrm{R} 5$ / Invest in bioinformatics skills and in silico approaches (recruitment of bio informaticians)

These two actions concern critical resources for deploying in silico approaches and exploiting their potential. Indeed, the we did not wish to comment on the various tools of "in silico", sharing the observation "of plenty of tools". The issue is more about the technical skills to be combined, in particular in bioinformatics and modeling. Several aspects were mentioned: the recruitment of bio informaticians in the healthcare establishments and research laboratories, the access to computing capacities orthe access to the skills and expertise of computer expert centers in certain major centers such as INRIA

\section{Several points were addressed on the issue of data}

i. seeking patient consent to access the individual clinical-biological data

ii. the opinion of the CNIL and the CCTIRS on the BCBs

iii. the organization of data sharing, with the system that will be implemented under the plan France Medicine Genomique 2025 as example

iv. the role that the cooperative structures can play in the constitution, management and administration of these BCBs,

v. the possibility of conducting experimental projects, pilot programmes, demonstrators

These new in silico approaches and the central place of individual patient data are at the heart of a profound transformation of clinical research. The third axis develops several proposals in this area.

The world of research and the health authorities at the federal and community level (FDA, EMA), but also INCA at the national level with "basket" or "umbrella" programmers have already initiated a shift in the design of clinical trials. This movement is global.

It is to R6/ Publicise and promote the "adaptive design" methods, through two vectors: a publication in a national journal (Bulletin du cancer for example), with a current situation report and the perspectives of approaches and new designs; a "white paper" on these new approaches. The goal is triple: to disseminate widely the existence of these new designs and to encourage the parties involved to make this changeover, to inform the health authorities of these new approaches, finally to seek to unite a community of researchers and clinicians around these new designs.

R9/ fully integrate real-life data into the continuous assessment of innovations

In these new designs, the place of real-life data is central. These data feed, on the one hand, the big data approaches; especially, they allow a real-life evaluation of the innovations, which are part of the continuity of the $\mathrm{R} \& \mathrm{D}$ process. There are many programmes and projects in this field, the challenge is to maintain the efforts in this direction and to achieve a level of statistical robustness that meets the requirements of the health authorities, hence the following proposal R6 ter/ Set up a research programme on the methodological elements 
of "adaptive design" to be consolidated (causal inference, recruitment bias, prescription bias, etc.) Several leads have been outlined; examples of action are provided for each of them (in parentheses, the "playful" post-it verbatim):

Modalities of registration of innovations: for a shorter process, multi-criteria end-points, the importance of RWD (Responsive web design): R6 bis/ Experiment multiple-industry collaborative trials in the early stages of development (pre-clinical, phase 0 ).

(A1: Transcelerate launches its 100th participatory clinical trial: 20 manufacturers have pooled their phase I molecules in 1,000 centres worldwide)

a) Focus the evaluation of clinical benefits on real-life data; consider the registration of innovations based on real-life data (A2: Engage a European programme (H2020) that is largely equipped on RWD: statistical methods (causal inference), close monitoring, connected objects, etc.; A3: The recording of the real-life evaluation of skin $\mathrm{K}$ [layers] has made it possible to pay the enormous progress made at the right price) (A4: Focus on randomised phase II trials for faster results)

b) Organise a more iterative and collaborative process of phases II and III, with active involvement of health authorities (A5: 30\% decrease in the social security deficit due to doctor - manufacturer collaborations)(A6: Europe finances new approaches to medical development: results in less than three years)

Approach to evaluation in the design of clinical trials; h: city-hospital assessment protocol->patientcentric (fewer evaluation criteria); i: what endpoint? $->$ clinicalbene fits for the patient = long-term temporalities

c) Use the potential of connected objects to collect real-time in vivo data and share them for clinical research purposes (C/I/G 1: new approach to drug evaluation: the reflection of your state of health, your watch tells us everything (QoL, biomarkers))

d) Standardize and homogenise these new designs in an international framework(E/F 1: A new ICH guideline for CTD of Clinical trials and pharmacoeconomic evaluation)

Include patients at earlier stages of the disease; G: trials with patients with cases as close as possible to the real life

A. B1: earlier clinical research to heal more

B. Use the digital tools for patient recruitment in clinical trials (G1: the e-trial mobile application allows the remote inclusion of its 100,000th patient)
C. Use the digital tools to establish clinical and biological bases that are full of patients and that facilitate to collection of individual consent to use the data for research purposes (or even donation of data) (G2: The K group (+ large social network of patients with $\mathrm{K}, 30$ million people) opens its shared data to open-data manufacturers)

Biomarkers: Toxicity, Efficacy, Iterative Biopsy, Adaptive Design

i. Develop the "biomarker" section in adaptive designs, including in real life (C1: Because of the new models of clinical trials, the 208,000th patient has just received the treatment adapted to their tumour, while taking into account the drug reaction in relation to the patient)

In silico : Target Identification / VirtualisationModelling; E: Importance Of Phases 0: Good Indicators For The Target Populations (Potential Risks)

a) D1: Arrival on the market of the first anti-cancer drug fully developed in silico

Through these leads, it is clear that the evolution of the design of clinical trials is not only an adaptation to a new medicine, which is that of precision medicine. It is also a question of correcting some biases of the current trials: recruitment bias, prescription bias, and causative inference for RWDs, difficulties in collecting real-life data, difficulties in sharing BCB data, importance of biological ration, etc.

The new designs of clinical trials profoundly change the R\&D process of innovations, which becomes more dynamic and interactive, less focused on the randomized phase III trials and building more and more bridges with the real-life data collected from observational studies (including connected objects) and cohorts. These change sare largely embraced worldwide by clinicians and drug manufacturers. This change is driven by the scientific and medical advances (in particular targeted immunotherapies) and by economic imperatives, which invalidate the assumption of keeping an RCT system for all the marketing authorization registration tests. Yet, while the EMA progressively integrates these new clinical research standards for the issuance of marketing authorizations, such movement should also be adopted by national health authorities, ANSM for France. The Transparency Committee appears to still keep a cautious stance with regard to the data from these newgeneration clinical trials, which should move in the future to actually take these data into account in the evaluation of innovations (clinical component of HBP The general idea is simple: to converge with the HAS on a methodological protocol that follows the evolution of the design of clinical trials and the concerns about data robustness. 


\section{R7/ Adapt The Assessment Methods Of The National}

Health Authorities To Take Into Account The Data of This New Generation Of Pragmatic And Adaptive Clinical Trials R8/ Open A New Early Conditional Access Path To The Market For The Most Promising Innovations

Arguments of these two proposals:

i. The clinical research has taken a global turn. Cancer care, with the targeted therapies, is at the forefront of this movement and one of its facets is the non-systemic nature of phase III randomized trials.

ii. This turn is sustainable, driven by the scientific progress and the impossibility of funding all the trials corresponding to all the possible therapeutic combinations.

iii. These new clinical trial designs, based on a more robust biological rationality and new statistical methodologies make it possible to obtain reliable results on the efficacy of innovations at an earlier stage of development, from the 2 nd phases (IIA, IIB).

iv. Many clinical trial investigators consider phase III trials to be a waste of time and money, and especially a loss of opportunity for their patients because they delay access to the innovations in the market, hence in medical care. However, these phases III trials are often conducted for industrial and commercial reasons (registration and bio statistical robustness for the manufacturer-payer negotiation and evaluation).

v. Clinicians recommend going directly from the probative phase II trials to real-life phases, with real-life evaluation of patients.

vi. Two consequences for the authorities: accept to consider phase II trials for the access procedure and integrate the real-life data for the revaluation.

vii. Clinicians recommend that HAS should provide for a procedure of conditional access to innovations (conditional reimbursement, conditional price).

\section{References}

1. Bates D W, Saria S, Ohno-Machado L, Shah A, Escobar G (2014) Big Data In Health Care: Using Analytics To Identify And Manage High-Risk And High-Cost Patients. Health Affairs 33(7): 1123-1131.

2. Dumez H, PrMinvielle E, Marrauld L Le (2015) Big Data en santé,Note complémentaire au rapport : Etat des lieux de l'innovation en santé numérique. Madame Laurie Marrauld p. 1-16.

3. Murdoch T B, Detsky A S (2013) The Inevitable Application of Big Data to Health Care, TheJama Network Avril 309(13): 1351-1352.

4. (2010) Guidance for Industry: Adaptive Design Clinical Trials for Drugs and Biologics. Biotechnology Law Report 29(2): 197-215.

5. Le biobanking, ou comment aider (très) facilement la recherche.

6. Maisonneuve H, Babany G (2015) Données de vie réelle et recherche clinique : le complément des données des essais randomisés ? Presse Med.

7. Mahajan R (2015) Real world data: Additional source for making clinical decisions. International Journal of Applied and Basic Medical Research 5(2): 82.

8. https://www.inpi.fr/fr/comprendre-la-propriete-intellectuelle/lebrevet.

9. http://www.wipo.int/sme/fr/documents/pharma_licensing.html

10. http://social-sante.gouv.fr/systeme-de-sante-et-medico-social/ recherche-et-innovation/france-genomique.

11.http://www.e-cancer.fr/Patients-et-proches/Se-faire-soigner/ Traitements/Therapies-ciblees-et-immunotherapie-specifique/ Acces-aux-nouveaux-traitements.

12.file:// / C: / Users /zingerf/Downloads / La \% 20 charte $\% 20$ AcS\%C3\%A9\%20(version\%20du\%2030\%20juin\%202016).pdf

13. https://www.inria.fr/

14.https://www.cnil.fr/

15. http://www.enseignementsup-recherche.gouv.fr/cid20537/cctirs. html

16. http://www.e-cancer.fr/Professionnels-de-la-recherche/Recherchetranslationnelle/Les-SIRIC

17. http://ansm.sante.fr/

18. http://www.has-sante.fr/portail/

\section{Your next submission with Juniper Publishers} will reach you the below assets

- Quality Editorial service

- Swift Peer Review

- Reprints availability

- E-prints Service

- Manuscript Podcast for convenient understanding

- Global attainment for your research

- Manuscript accessibility in different formats

( Pdf, E-pub, Full Text, Audio)

- Unceasing customer service

Track the below URL for one-step submission _ https://juniperpublishers.com/online-submission.php 